\title{
Biomechanically Driven Registration of Pre- to Intra-Operative 3D Images for Laparoscopic Surgery
}

\author{
Ozan Oktay $^{1}$, Li Zhang ${ }^{1}$, Tommaso Mansi ${ }^{1}$, Peter Mountney ${ }^{1}$, Philip Mewes ${ }^{2}$, \\ Stéphane Nicolau ${ }^{3}$, Luc Soler ${ }^{3}$, and Christophe Chefd'hotel ${ }^{1}$ \\ 1 Siemens Corporation, Corporate Technology, Princeton, NJ, USA \\ 2 Siemens AG, Healthcare AX, Forchheim, Germany \\ 3 IRCAD, Virtual-Surg, Place de l'Hospital 1, 67091 Strasbourg Cedex, France
}

\begin{abstract}
Minimally invasive laparoscopic surgery is widely used for the treatment of cancer and other diseases. During the procedure, gas insufflation is used to create space for laparoscopic tools and operation. Insufflation causes the organs and abdominal wall to deform significantly. Due to this large deformation, the benefit of surgical plans, which are typically based on pre-operative images, is limited for real time navigation. In some recent work, intra-operative images, such as cone-beam CT or interventional CT, are introduced to provide updated volumetric information after insufflation. Other works in this area have focused on simulation of gas insufflation and exploited only the pre-operative images to estimate deformation. This paper proposes a novel registration method for pre- and intra-operative 3D image fusion for laparoscopic surgery. In this approach, the deformation of pre-operative images is driven by a biomechanical model of the insufflation process. The proposed method was validated by five synthetic data sets generated from clinical images and three pairs of in vivo CT scans acquired from two pigs, before and after insufflation. The results show the proposed method achieved high accuracy for both the synthetic and real insufflation data.
\end{abstract}

\section{Introduction}

Laparoscopic surgery is a widely used minimally invasive procedure for cancer and other disease treatment. Advantages like reduced post-operative pain and shorter recovery time make it preferable to open surgery. During surgery, the laparoscopic camera can only visualize the surface of the tissue this makes localizing sub surface structures, such as vessels and tumors, challenging. Therefore, intra-operative 3D images are introduced to provide updated information. While the intra-operative images typically have limited image information due to the constraints imposed in operating rooms, the pre-operative images can provide supplementary anatomical and functional details, and carry accurate segmentation of organs, vessels, and tumors. To bridge the gap between surgical plans and laparoscopic images, registration of pre- and intra-operative 3D images is needed to guide real time navigation. However, this registration is challenging due to 
gas insufflation [1] and other surgical preparation [2, which result in large organ deformation and sliding between viscera and abdominal wall (Fig. 11). Therefore, a standard non-rigid registration method cannot be directly applied, and the topic has not been investigated much in depth in literature.
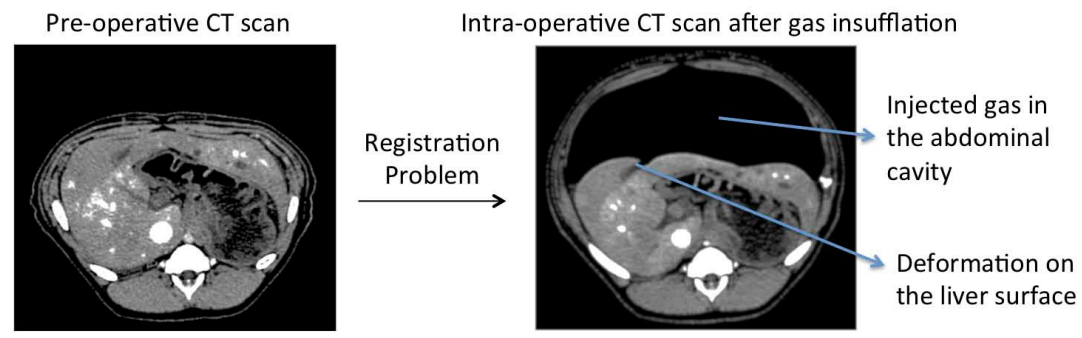

Fig. 1. Due to large deformations, standard registration methods like FEM and dense diffeomorphic registration, fail to achieve accurate structure alignment

Recent research has focused on insufflation modeling using only pre-operative images and registration of pre-operative images to laparoscopic images [34. However, none of the proposed methods have made use of the intra-operative images in their framework. For example, Kitasaka et al. [3] use elastic deformation model to simulate the anatomical changes caused by pneumoperitoneum. In their work, artificial gas pressure is simulated with a biomechanical model in abdominal cavity. More recently, Bano et al. [56] simulate the gas insufflation by lifting the abdominal wall in pre-operative images. Specifically, in [5], the deformation model is simulated using finite element method (FEM) to compute the displacements. In [6], the displacements are found by matching the landmarks collected from patient's insufflated abdominal surface. Although the proposed deformation models are physically realistic, the models are not guided by the available target, intra-operative images. In fact, the intensity information in both images could be used together with the gas insufflation model in a registration algorithm.

In this paper, a method is proposed for accurate registration of pre-operative images to intra-operative images driven by biomechanical modeling of abdomen deformation under gas insufflation. The coupling between the registration and insufflation model is achieved by optimizing the intensity similarity measure between the modeled pre-operative image and the intra-operative image. The dynamic model parameter optimization with intensity information differs our approach from the existing methods 35 , that used constant model parameters.

\section{Method}

The registration method consists of two main steps: insufflation modeling and diffeomorphic non-rigid registration. The first step computes the deformations and organ shifts caused by gas pressure, using the biomechanical model, which is 
based on the mechanical parameters and pressure level. This model is applied on pre-operative images to achieve an initial alignment with intra-operative images, which accounts for both non-rigid and rigid transformations caused by the insufflation. The biomechanical model is incorporated into the registration framework by coupling the model parameters with an intensity similarity measure unlike the models in [35, and the finite element method (FEM) registration methods in 78. In the second part, diffeomorphic registration, which has a higher degree of freedom, refines the surface differences between the pre-operative image, warped according to the biomechanical model, and the intra-operative image. The proposed method is shown in Fig. 2 .

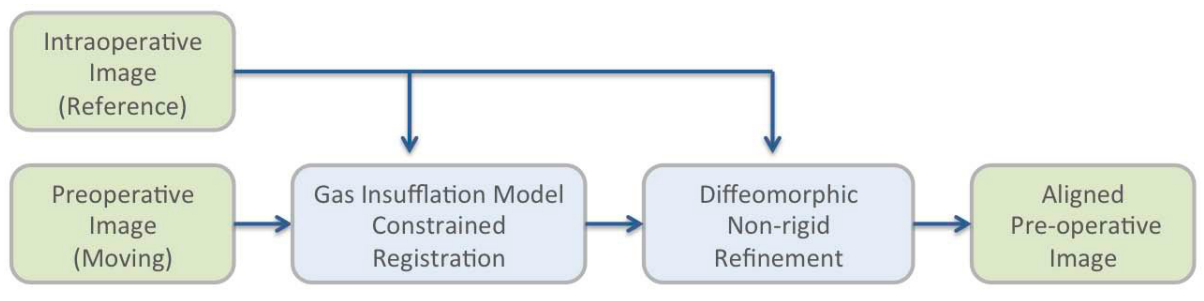

Fig. 2. Diagram of the proposed method

Initial image alignments are done using spine position [9], when the images are separated. The optimization problem can be formulated as follows:

$$
\max D^{\mathrm{LCC}}\left(\mathbf{R}, \Psi_{d} \circ \Psi_{m} \circ \mathbf{M}\right)
$$

where $\mathbf{M}$ and $\mathbf{R}$ denote the moving (pre-op) and reference 3D images (intra-op) respectively, and $D^{\mathrm{LCC}}$ is the local cross correlation intensity similarity measure. The model guided registration and diffeomorphic transformation are represented with $\Psi_{m}$ and $\Psi_{d}$ respectively.

\section{Insufflation Model}

The biomechanical model deforms mesh elements and computes the displacement of mesh points. In the proposed framework the pre-operative image is segmented as liver, abdominal wall and surrounding tissue using a semi-automated method, and tetrahedral mesh elements are generated using CGAI1 . Similarly, automatic and manual segmentation tools can be used as well since this task is performed before surgery. Next, surface points of these volume meshes are tagged automatically, which are used to apply the insufflated gas pressure.

A corotational finite element method is used with a linear elastic model to compute the displacement of the organs under abdominal gas pressure. An implicit time discretization is employed for unconditional stability. As a solution method, dynamic modeling is selected to cope with inertia effects and organ

1 WWW.cgal.org 


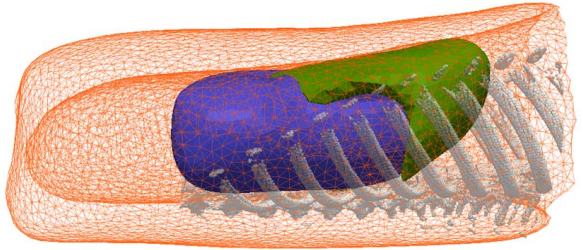

(a) Initial mesh before the insufflation

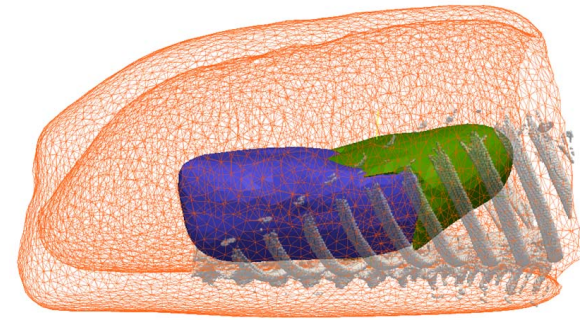

(b) Deformed mesh after the insufflation

Fig. 3. Abdominal wall (red), liver (green), and surrounding tissues (blue) are deformed with the proposed registration method based on gas insufflation model

collisions. The mechanical system with abdominal wall, liver, and surrounding tissues is deformed under the internal pressure generated in the abdominal cavity, which represents the insufflated gas, shown in Fig. 3 The applied force field together with the internal tissue properties deform the meshes while preserving the mesh topology. The force, acceleration, and displacement field for each node element are integrated and computed in an iterative approach.

$$
M \ddot{u}+D \dot{u}+K u=F_{g a s}
$$

In (2), the partial differential equation for the mechanical model is given. When the model achieves stability, the gas pressure forces $\left(F_{\text {gas }}\right)$ are balanced with internal mesh stiffness forces, and the final node positions $(u)$ are determined. The parameters $M, D$, and $K$ represent the mass, damping, and stiffness matrices. In the proposed system, the model parameters are the gas pressure, Poisson's ratio, and Young's modulus for each tissue. The initial parameter values are set according to the literature [10]11, and the gas pressure value is collected during surgery. The framework has been implemented in SOFA2 library.

\section{Insufflation Model Constrained Registration}

The biomechanical gas insufflation model is combined with intensity similarity measure to include it in the registration framework. In this regard, the model parameters are updated iteratively until model convergence by optimizing the intensity similarity between the intra-operative and model updated pre-operative images. The deformation field obtained from the biomechanical model is used to recompute the pre-operative image that uses a backward thin-plate spline interpolation method. The algorithm can be described as follows: let the model deformations $\Psi_{m}: \mathbb{R}^{3} \rightarrow \mathbb{R}^{3}$ and mechanical parameter set $\alpha^{(t)} \in \mathbb{R}^{L}$ be denoted by $\Psi_{m, \alpha^{(t)}}$. The superscript $t$ is the discrete time step, and $L \in \mathbb{N}$ is the number of parameters. The similarity measure is maximized using gradient ascent approach, which updates the model parameter values.

\footnotetext{
2 http://www. sofa-framework.org
} 
Algorithm 1 summarizes the steps of the method, where $N, \delta$, and $\gamma$ are the number of mesh elements, parameter increment, and constant gradient step. The mechanical model is denoted by $F\left(\alpha^{(t)}, \mathbf{M}\right)$, and it is computed until model convergence, given in (2). Initially, the two images are aligned using rigid registration based on spine positions.

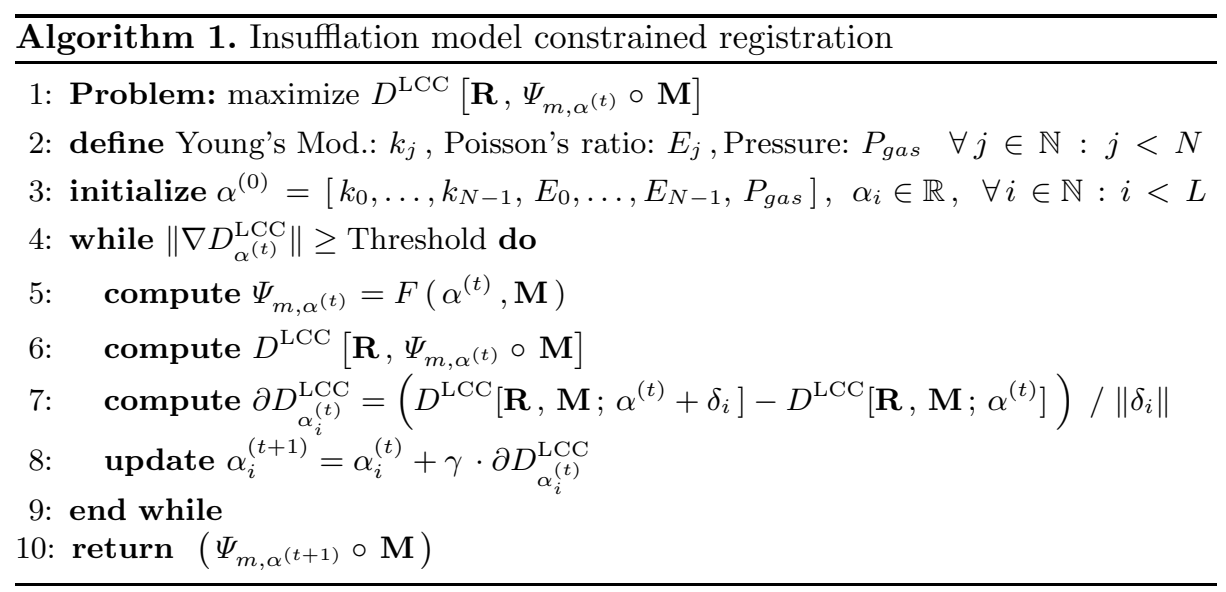

\section{Diffeomorphic Non-rigid Registration}

Tissue characteristics used to parameterize the biomechanical model are not patient specific and cannot comprehensively estimate the subtle complex deformations in the abdominal cavity or cardiac/respiration phase specific deformation. This results in a small amount of residual deformation. In this framework a refinement step is proposed to account for this residual deformation.

The model based registered pre-operative image is warped to intra-operative image using diffeomorphic non-rigid registration [12]. It is a dense matching method driven by the gradient of local cross correlation similarity measure. The Gaussian smoothing regularization is applied on time-dependent velocity fields and their composition is used to estimate deformations.

\section{Results}

The proposed gas insufflation model constrained registration approach was firstly evaluated on five different pairs of synthetic 3D-CT human abdominal images. The images were acquired from five different patients in a non-insufflated state by scanning the patients at two different time instances. The synthetic insufflated images were computed by applying the proposed insufflation model on one of the two images from each patient, with the mechanical parameter values provided in [5]. Additionally, for each pair, different Gaussian noise levels and downsampling were applied to assess the robustness of the method against these conditions, the details are provided in Table 11. The bar plot in Fig. 4 demonstrates that the method has registration error less than $2.5 \mathrm{~mm}$ even under large 
Table 1. Noise and downsampling conditions for intra-operative synthetic images

\begin{tabular}{lccccc} 
& Case 1 & Case 2 & Case 3 & Case 4 & Case 5 \\
\hline Noise level (st. deviation) & 0 & $\pm 70 \mathrm{HU}$ & $\pm 100 \mathrm{HU}$ & $\pm 100 \mathrm{HU}$ & $\pm 100 \mathrm{HU}$ \\
Downsample Ratio (x, y, z) & - & - & - & $(2,2,2)$ & $(2,2,2)$ \\
\hline
\end{tabular}

noise disturbance and low resolution conditions. The error values were obtained with mesh to mesh distance metric for both abdominal wall and liver surfaces.

The method was also validated on the real scenario by using three different pairs of insufflated and non-insufflated pig abdominal 3D-CT scans. The first two pairs were collected from two different pigs under full inspiration and contrast enhancement conditions. In the last pair (pig pair-3), the first pig was scanned with insufflation and half expiration, and registered with the full inspiration noninsufflated scan of the same pig. The quantitative assessment was done again using surface mesh to mesh distance. To demonstrate the contribution of the method, the same datasets were also registered using only diffeomorphic nonrigid registration. The ground-truth surface meshes were collected from intraoperative images using manual segmentation. The quantitative results and the alignment of the first pair are provided in Table 2 and Fig. 5 .

The mean distance error on the in vivo pig images was recorded as $0.88 \mathrm{~mm}$ and $1.75 \mathrm{~mm}$ for the abdominal wall and liver. It can be concluded that the proposed method gives better registration accuracy compared to biomechanical model alone approaches [5] that achieved an average registration error of $3.3 \mathrm{~mm}$ and $4.3 \mathrm{~mm}$ with the same validation method for skin and abdominal viscera. Furthermore, comparison against the method with only non-rigid registration approaches shows the contribution of including the insufflation model in registration framework. The computation time for the complete registration process

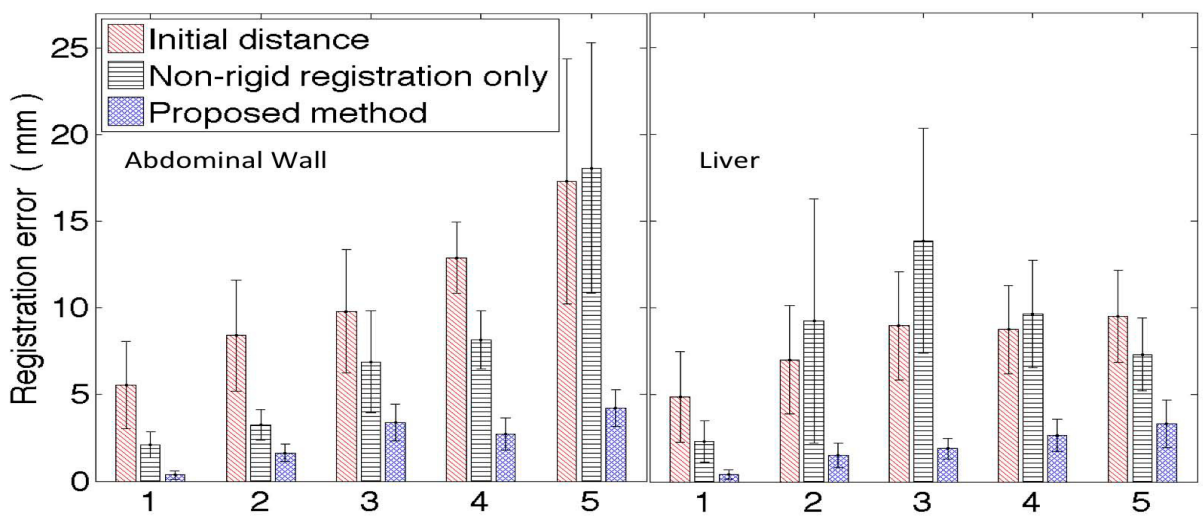

Fig. 4. Registration errors for synthetic images, generated from clinical data 
Table 2. Registration error for abdominal wall (A) and liver (L) of the pig data (mesh to mesh distance in $\mathrm{mm}$ ). I, D, and $\mathrm{M}$ denote the initial mesh distance, the distance error with only non-rigid registration, and the proposed method

\begin{tabular}{|c|c|c|c|c|c|c|c|c|c|}
\hline \multirow[b]{2}{*}{ Method } & \multicolumn{3}{|c|}{ Pig pair - 1} & \multicolumn{3}{|c|}{ Pig pair - 2} & \multicolumn{3}{|c|}{ Pig pair - 3} \\
\hline & I & $\mathrm{D}$ & $\mathrm{M}$ & $\mathrm{I}$ & $\mathrm{D}$ & $\mathrm{M}$ & I & $\mathrm{D}$ & $\mathrm{M}$ \\
\hline (A) Mean Err. & 11.42 & 12.63 & 0.67 & 8.74 & 7.08 & 0.84 & 12.56 & 12.69 & 1.13 \\
\hline (A) Max Err. & 64.16 & 77.45 & 2.64 & 41.76 & 61.85 & 3.59 & 65.85 & 88.38 & 3.98 \\
\hline (L) Mean Err. & 7.72 & 3.46 & 0.92 & 12.92 & 9.94 & 3.28 & 6.49 & 6.22 & 1.06 \\
\hline (L) Max Err. & 25.43 & 17.56 & 2.76 & 37.08 & 50.27 & 8.04 & 22.52 & 26.01 & 6.36 \\
\hline
\end{tabular}

was measured as 12 minutes per 3D image pair (Intel Xeon W3530 2.80GHz), which can be reduced to a few minutes through parallel optimization.

\section{Discussion and Conclusion}

In this paper, a method has been proposed to estimate the deformation caused after gas insufflation using pre- and intra-operative images. The validation of the proposed approach was performed on both synthetic human CT and in vivo pig CT scans. In the synthetic case, under noise and downsampling conditions, a mean registration error of less than $2.5 \mathrm{~mm}$ was obtained, which demonstrated the robustness of the method against noise disturbance and low resolution. In the second evaluation, insufflated and non-insufflated in vivo pig CT images were used. A mean registration error of $0.88 \mathrm{~mm}$ for liver and $1.75 \mathrm{~mm}$ for abdominal wall showed the applicability of the method in laparoscopic surgeries as

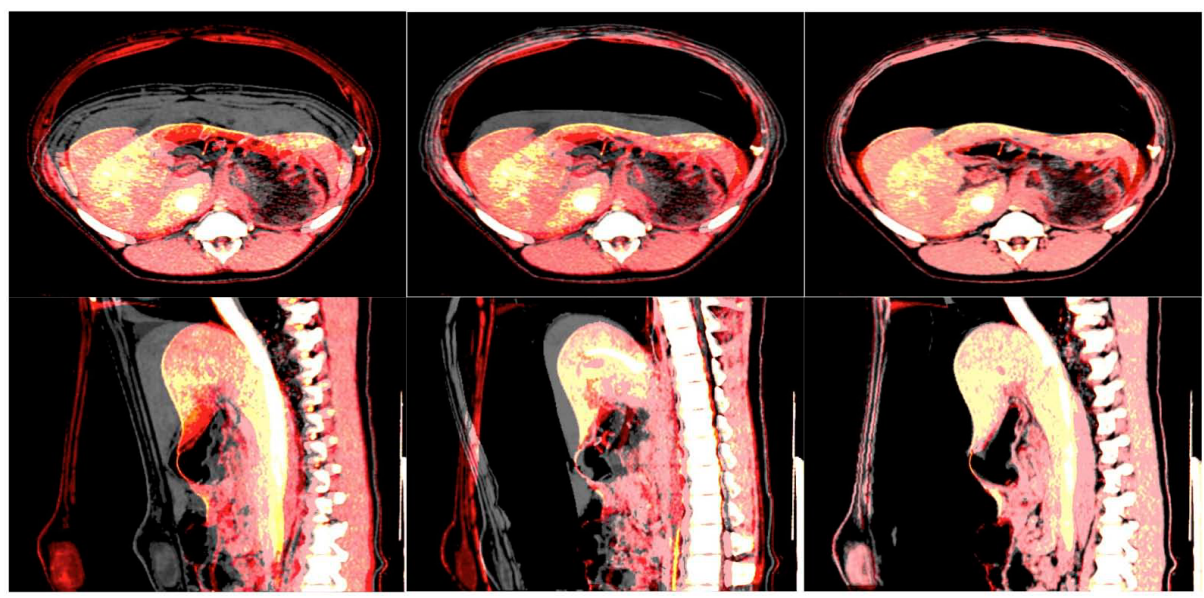

Fig. 5. Insufflated(red) and non-insufflated(gray) pig images after each step: initial position, model based registration, and diffeomorphic non-rigid refinement 
a registration algorithm. Furthermore, the error values were compared against the ones obtained with standard diffeomorphic non-rigid registration method, demonstrating the main contribution of the proposed method. Additionally, the error values showed that the use of registration approaches on estimating the gas insufflation deformations can provide more precise tissue alignment compared to only biomechanical model based approaches [5]. The proposed approach is generic; as such, it can be applied to other medical problems where large deformations appear due to external intervention (e.g. brain-shift, histology needle guidance). The future work will investigate: validity of the method on these problems and the effect of respiration and cardiac motion on the algorithm accuracy. Lastly, the authors thank Prof. J. Marescaux, Dr. A. Hostettler, IRCAD France \& Taiwan, and Show-Chwan Memorial Hospital for providing the pig data.

\section{References}

1. Zijlmans, M., Langø, T., Hofstad, E., Van Swol, C., Rethy, A.: Navigated laparoscopy-liver shift and deformation due to pneumoperitoneum in an animal model. Minimally Invasive Therapy \& Allied Technologies 21(3), 241-248 (2012)

2. Heizmann, O., Zidowitz, S., Bourquain, H., Potthast, S., Peitgen, H., Oertli, D., Kettelhack, C.: Assessment of intraoperative liver deformation during hepatic resection: prospective clinical study. World Journal of Surgery 34(8), 1887-1893 (2010)

3. Kitasaka, T., Mori, K., Hayashi, Y., Suenaga, Y., Hashizume, M., Toriwaki, J.i.: Virtual pneumoperitoneum for generating virtual laparoscopic views based on volumetric deformation. In: Barillot, C., Haynor, D.R., Hellier, P. (eds.) MICCAI 2004. LNCS, vol. 3217, pp. 559-567. Springer, Heidelberg (2004)

4. Davatzikos, C., Shen, D., Mohamed, A., Kyriacou, S.K.: A framework for predictive modeling of anatomical deformations. IEEE Transactions on Medical Imaging 20, 836-843 (2001)

5. Bano, J., et al.: Simulation of pneumoperitoneum for laparoscopic surgery planning. In: Ayache, N., Delingette, H., Golland, P., Mori, K. (eds.) MICCAI 2012, Part I. LNCS, vol. 7510, pp. 91-98. Springer, Heidelberg (2012)

6. Bano, J., Hostettler, A., Nicolau, S., Doignon, C., Wu, H.S., Huang, M.H., Soler, L., Marescaux, J.: Simulation of the abdominal wall and its arteries after pneumoperitoneum for guidance of port positioning in laparoscopic surgery. In: Bebis, G., et al. (eds.) ISVC 2012, Part I. LNCS, vol. 7431, pp. 1-11. Springer, Heidelberg (2012)

7. Ferrant, M., Warfield, S.K., Guttmann, C.R.G., Mulkern, R.V., Jolesz, F.A., Kikinis, R.: 3D image matching using a finite element based elastic deformation model. In: Taylor, C., Colchester, A. (eds.) MICCAI 1999. LNCS, vol. 1679, pp. 202-209. Springer, Heidelberg (1999)

8. Schnabel, J.A., Tanner, C., Castellano-Smith, A.D., Degenhard, A., Leach, M.O., Hose, R., Hill, D.L.G., Hawkes, D.J.: Validation of nonrigid image registration using finite-element methods: application to breast MR images. IEEE Transactions on Medical Imaging 22(2), 238-247 (2003)

9. Zhang, L., Chefd'hotel, C., Ordy, V., Zheng, J., Deng, X., Odry, B.: A knowledgedriven quasi-global registration of thoracic-abdominal CT and CBCT for imageguided interventions. In: SPIE Medical Imaging, p. 867110 (2013) 
10. Song, C., Alijani, A., Frank, T., Hanna, G.B., Cuschieri, A.: Mechanical properties of the human abdominal wall measured in vivo during insufflation for laparoscopic surgery. Surgical Endoscopy 20(6), 987-990 (2006)

11. Samur, E., Sedef, M., Basdogan, C., Avtan, L., Duzgun, O.: A robotic indenter for minimally invasive characterization of soft tissues. In: International Congress Series, vol. 1281, pp. 713-718 (2005)

12. Chefd'Hotel, C., Hermosillo, G., Faugeras, O.D.: Flows of diffeomorphisms for multimodal image registration. In: ISBI, pp. 753-756 (2002) 